\title{
COMPACTNESS IN $L^{2}$ AND THE FOURIER TRANSFORM
}

\author{
ROBERT L. PEGO
}

\begin{abstract}
The Riesz-Tamarkin compactness theorem in $L^{p}\left(\mathbf{R}^{n}\right)$ employs notions of $L^{p}$-equicontinuity and uniform $L^{p}$-decay at $\infty$. When $1 \leqslant p \leqslant 2$, we show that these notions correspond under the Fourier transform, and establish new necessary and sufficient criteria for compactness in $L^{2}\left(\mathbf{R}^{n}\right)$.
\end{abstract}

An oft-quoted classical result characterizing compact sets in $L^{p}\left(\mathbf{R}^{n}\right)$ is due to M. Riesz and J. D. Tamarkin (see [1, 2, 4]):

THEOREM. $A$ bounded subset $K$ of $L^{p}\left(\mathbf{R}^{n}\right), 1 \leqslant p<\infty$, is conditionally compact if and only if

(I) $\int_{\mathbf{R}^{n}}|f(x+y)-f(x)|^{p} d x \rightarrow 0$ as $y \rightarrow 0$ uniformly for $f$ in $K$, and

(II) $\int_{|x|>R}|f(x)|^{p} d x \rightarrow 0$ as $R \rightarrow \infty$ uniformly for $f$ in $K$.

Property (I) is a uniform smoothness property. By analogy with the terminology of Arzela-Ascoli, we say the functions in $K$ are $L^{p}$-equicontinuous if (I) holds. Property (II) is a uniform decay property. The connection between smoothness and decay through the Fourier transform has been well explored [6]. Yet the following nice equivalence seems to be new:

THEOREM 1. Let $K$ be a bounded subset of $L^{2}\left(\mathbf{R}^{n}\right)$ and let $\hat{K}$ be the Fourier transform of $K, \hat{K}=\{\hat{f} \mid f \in K\}$. The functions of $K$ are $L^{2}$-equicontinuous if and only if the functions of $\hat{K}$ decay uniformly in $L^{2}$, and vice versa. That is, $K$ satisfies $(\mathrm{I})$ in $L^{2}$ if and only if $\hat{K}$ satisfies (II) in $L^{2}$, and vice versa.

Combining this result with the Riesz-Tamarkin theorem, we obtain two alternative characterizations of compact sets in $L^{2}\left(\mathbf{R}^{n}\right)$ :

THEOREM 2. $A$ bounded subset $K$ of $L^{2}\left(\mathbf{R}^{n}\right)$ is conditionally compact if and only if $\int|f(x+y)-f(x)|^{2} d x \rightarrow 0$ as $y \rightarrow 0$, and $\int|\hat{f}(\xi+\omega)-\hat{f}(\xi)|^{2} d \xi \rightarrow 0$ as $\omega \rightarrow 0$, both uniformly for $f$ in $K$.

THEOREM 3. A bounded subset $K$ of $L^{2}\left(\mathbf{R}^{n}\right)$ is conditionally compact if and only if $\int_{|x|>R}|f(x)|^{2} d x \rightarrow 0$ and $\int_{|\xi|>R}|\hat{f}(\xi)|^{2} d \xi \rightarrow 0$ as $R \rightarrow \infty$, both uniformly for $f$ in $K$.

Received by the editors November 7, 1984.

1980 Mathematics Subject Classification. Primary 46E30, 42B99.

Key words and phrases. Compactness, Fourier transform, $L^{p}, L^{2}, L^{p}$-equicontinuity. 
Theorem 1 is an easy consequence of the theorem below, which offers some results in $L^{p}, 1 \leqslant p \leqslant 2$.

THEOREM 4. Let $K$ be a bounded subset of $L^{p}, 1 \leqslant p \leqslant 2$. If $K$ satisfies (I) (resp. (II)) in $L^{p}$, then $\hat{K}$ satisfies (II) (resp. (I)) in $L^{q}$, where $1 / p+1 / q=1$. (If $q=\infty$, conditions (I) and (II) are to be stated in the obvious way using the sup norm.)

Let us set our notation and recall some basic results. For $f \in L^{1}\left(\mathbf{R}^{n}\right)$,

$$
\hat{f}(\xi)=\int_{R^{n}} f(x) e^{-i \xi \cdot x} d x .
$$

Recall [3]:

(1) The Fourier transform above extends to a bounded linear map $f \rightarrow \hat{f}$ from $L^{p}$ to $L^{q}$, for $1 \leqslant p \leqslant 2$ and $1 / p+1 / q=1$, so $\|\hat{f}\|_{q} \leqslant C_{p}\|f\|_{p}$ for $f$ in $L^{p}$.

(2) For $f$ in $L^{p}, \omega$ in $\mathbf{R}^{n}$, we have $\left[e^{-i \omega x} f(x)\right]^{\wedge}(\xi)=\hat{f}(\xi+\omega)$ in $L^{q}$.

(3) For $f$ in $L^{p}, \psi$ in the Schwartz class $\mathscr{S},(f * \psi) \hat{)}(\xi)=\hat{f}(\xi) \hat{\psi}(\xi)$ in $L^{q}$, where $f * \psi(x)=\int_{\mathbf{R}^{n}} f(x-y) \psi(y) d y$.

Proof of Theorem 4. First, we assume $K$ satisfies (II) in $L^{p}$. Let $M$ be a bound for $K$ in $L^{p}$. For $f$ in $K$,

$$
\hat{f}(\xi+\omega)-\hat{f}(\xi)=\left[\left(e^{-i \omega \cdot x}-1\right) f(x)\right]^{\wedge}(\xi),
$$

whence

$$
\begin{aligned}
\|\hat{f}(\xi+\omega)-\hat{f}(\xi)\|_{q} & \leqslant C_{p}\left\|\left(e^{-i \omega \cdot x}-1\right) f(x)\right\|_{p} \\
& \leqslant C_{p}\left(\int_{|x| \leqslant R}(|\omega||x||f(x)|)^{p} d x+2 \int_{|x|>R}|f(x)|^{p} d x\right)^{1 / p} .
\end{aligned}
$$

Let $\varepsilon>0$. Because of (II) we may choose $R$ so large that the second term here is less than $\frac{1}{2}\left(\varepsilon / C_{p}\right)^{p}$ independent of $f$ in $K$. Then since $\int_{|x| \leqslant R}(|x||f(x)|)^{p} d x \leqslant(R M)^{p}$ for $f$ in $K$, we have $\|\hat{f}(\xi+\omega)-\hat{f}(\xi)\|_{q}<\varepsilon$ if $\omega$ is sufficiently small, $|\omega|^{p}$ $<\frac{1}{2}\left(\varepsilon / C_{p} R M\right)^{p}$, independent of $f$ in $K$. So $\hat{K}$ satisfies (I) in $L^{q}$.

Now assume $K$ satisfies (I) in $L^{p}$. We seek to show that functions in $\hat{K}$ decay uniformly in $L^{q}$. Let $\psi(x)=(2 \pi)^{-n / 2} e^{-|x|^{2} / 2}, \psi_{R}(x)=\psi(R x) R^{n}$, so that $\psi_{R}$ and $\hat{\psi}_{R}(\xi)=\hat{\psi}(\xi / R)$ are in $\mathscr{S}$, with $\hat{\psi}(\xi)=e^{-|\xi|^{2} / 2}, \hat{\psi}_{R}(0)=\int \psi_{R}(y) d y=1$. Now for $|\xi| \geqslant 2 R, \frac{1}{2} \leqslant 1-\hat{\psi}_{R}(\xi)$, so for $f \in K$,

$$
\begin{aligned}
\frac{1}{2}\left(\int_{|\xi|>2 R}|\hat{f}(\xi)|^{q} d \xi\right)^{1 / q} & \leqslant\left\|\hat{f}(\xi)\left(1-\hat{\psi}_{R}(\xi)\right)\right\|_{q} \\
& \leqslant C_{p}\left\|f(x)-f * \psi_{R}(x)\right\|_{p} \\
& =C_{p}\left[\int\left|\int(f(x)-f(x-y)) \psi_{R}(y) d y\right|^{p} d x\right]^{1 / p} .
\end{aligned}
$$

By Jensen's inequality and Fubini's theorem, this is

$$
\leqslant C_{p}\left[\int\left[\int\left|f(x)-f\left(x-\frac{y}{R}\right)\right|^{p} d x\right] \psi(y) d y\right]^{1 / p} \text {. }
$$


Now define a uniform $L^{p}$ modulus of continuity for $K$,

$$
H(y)=\sup _{f \in K} \int|f(x)-f(x-y)|^{p} d x .
$$

By (I), $H(y) \rightarrow 0$ as $y \rightarrow 0$, and $H(y) \leqslant(2 M)^{p}$ for all $y$. From above, we have

$$
\left[\int_{|\xi|>2 R}|\hat{f}(\xi)|^{q} d \xi\right]^{1 / q} \leqslant 2 C_{p}\left[\int H\left(\frac{y}{R}\right) \psi(y) d y\right]^{1 / p} \rightarrow 0
$$

as $R \rightarrow \infty$ uniformly for $f$ in $K$. Hence, $\hat{K}$ satisfies (II).

We conclude with a small application, which illustrates a principle known in information theory (see [5]) that an operator in $L^{2}$ that is "band limited and time limited" is compact.

Fix any $\phi_{1}(x), \phi_{2}(x)$ bounded functions on $\mathbf{R}^{n}$ which satisfy $\lim _{|x| \rightarrow \infty} \phi_{i}(x)=0$, $i=1,2$, and let $\phi_{i}$ denote the multiplication operator on $L^{2}$ given by $u(x) \rightarrow$ $\phi_{i}(x) u(x), i=1,2$. Let $F$ denote the Fourier transform operator $u \rightarrow F u=\hat{u}$. Define an operator $T$ on $L^{2}$ by $T=\phi_{1} F \phi_{2}$. Assume $\phi_{1}(x)$ is continuous.

Proposition. $T$ is a compact operator on $L^{2}$.

Proof. Let $K$ be a bounded set in $L^{2}$. Clearly, the set $\phi_{2} K$ has the uniform decay property (II) in $L^{2}$. From Theorem 1 , the set $F \phi_{2} K$ is $L^{2}$-equicontinuous (has property (I)). The set $T K=\phi_{1} F \phi_{2}$ is also $L^{2}$-equicontinuous, and also has the uniform decay property (II). By Riesz-Tamarkin, it follows that $T K$ is precompact. Q.E.D.

ACKNOWLEDGEMENT. The author thanks Jonathan Goodman for pointing out this application.

\section{REFERENCES}

1. R. Adams, Sobolev spaces, Academic Press, New York, 1975.

2. N. Dunford and J. T. Schwartz, Linear operators, Part I, Wiley-Interscience, New York, 1966.

3. E. M. Stein and G. Weiss, Introduction to Fourier analysis on Euclidean spaces, Princeton Univ. Press, Princeton N. J., 1971.

4. K. Yosida, Functional analysis, 2nd ed., Springer-Verlag, New York, 1968.

5. H. J. Landau and H. O. Pollack, Prolate spherodial wave functions, Fourier analysis, and uncertainty. III, Bell System Tech. J. 41 (1962), 1295-1336.

6. M. Murata, A theorem of Liouville type for partial differential equations with constant coefficients, J. Fac. Sci. Univ. Tokyo IA 21 (1974), 395-404.

Department of Mathematics, University of Michigan, Ann Arbor, Michigan 48109 